\title{
Treatment Result Following Fluoroscopic Guided Close Reduction and Internal Fixation by Per-Cutaneous Crossed K-Wires of Extension Type Gartland Type-II and Type-III Supracondylar Fracture of Humerus in Children
}

Tafhim Ehsan Kabir ${ }^{{ }^{*}}$

A.N.M Humayun Kabir ${ }^{1}$

Alak Kanti Biswas $^{1}$

Rahma Binte Anwar ${ }^{2}$

Touhidul Islam ${ }^{1}$

Tusher Kanti Nath

${ }^{1}$ Department of Orthopedics

Chattogram Maa-O-Shishu Hospital Medical College Chattogram, Bangladesh.

${ }^{2}$ Department of Anaesthesia

Chittagong Medical College Hospita

Chattogram, Bangladesh.
*Correspondence to:

Dr Tafhim Ehsan Kabir

Associate Professor

Department of Orthopedics

Chattogram Maa-O-Shishu Hospital Medical College

Chattogram, Bangladesh.

Mobile : +88 1712276487

Email : tafhimekf@yahoo.com

Date of Submission ： 20.04 .2021

Date of Acceptance : 08.06 .2021

www.banglajol.info/index.php/CMOSHMCJ

\begin{abstract}
Background : Supracondylar fractures of the humerus is one of the most common fractures in children. Failure to treat properly leads to malunion of the fracture site. For that, closed reduction and percutaneous pinning is considered to be the golden choice for treatment. There are many methods in which percutaneous $k$-wire fixation can be done. The aim of this study is to report the advantages of percutaneous fixation using cross pinning from both medial and lateral sides.
\end{abstract}

Materials and methods : A total number of seventy pediatric patients with Gartland type II and type III supracondylar fracture of the humerus were recruited from the outpatient department of two different hospitals between January 2018 and September 2020. All of them were treated using closed reduction and internal fixation using percutaneous crossed $k$-wires. The treatment outcomes were evaluated using Flynn's criteria and were compared with other similar studies.

Results : The mean age of study subjects was $8.14 \pm 2.8$ and the male to female ratio was 1.6:1. In 27(38.6\%) cases the left arm was involved while in 43(61.4\%) cases the right arm was involved. Preoperative complications included $1(1.4 \%)$ case with radial nerve palsy and $4(5.7 \%)$ cases with pulseless pink hand. When evaluating cosmetic outcome using Flynn's criteria, there were 57(81.4\%) excellent, $10(14.3 \%)$ good and 3(4.3\%) fair outcomes. On evaluating outcome according to range of motion deficit outcomes were excellent in 35(50\%), good in 22(31.4\%), fair in $5(7.2 \%)$ and poor in $8(11.4 \%)$ children. Post-operative complications were $1(1.4 \%)$ ulnar nerve neuropraxia and 5(7.2\%) superficial pin tract infections.

Conclusion : Closed reduction and internal fixation using percutaneous crossed $k$ wires placed from the medial and lateral side gives satisfactory cosmetic and functional outcomes in majority of the patients with Gartland type II and III supracondylar fractures of the humerus.

Key words : Close reduction; Internal fixation; K-wires; Supracondylar fracture.

\section{INTRODUCTION}

Supracondylar fracture of the humerus is one of the most common fractures encountered in the pediatric population ${ }^{1,2}$. These fractures usually occur when a child falls on an outstretched hand while the elbow is in full extension. Mostly these fractures are of extension type and are classified according to the classification system by Gartland which guides the standard of care for treatment of this entity ${ }^{3}$. This classification is based on the degree and direction of displacement along with the presence or absence of an intact cortex ${ }^{4}$. In Gartland type I fracture the bones on either ends of the fracture site are undisplaced or minimally displaced. In Gartland type II fractures, the posterior cortex is intact and angulation is present. In other words, the fracture is displaced, but the cortex remains in contact. In Type III 
fractures, there is complete displacement with no bony hinge and the distal fragment is displaced posteriorly. The preferred treatment for such displaced fractures is closed reduction and percutaneous crossed Kirschner wire (K-wire) fixation. This additional fixation helps maintain the reduction until bony union hence preventing late collapse of fracture site and providing better results ${ }^{5}$.

Since most treatments are associated with some known risks, contradictions vary widely based on the treatment administered. One such particular concern when managing supracondylar fractures of the humerus is the potential of this fracture to cause vascular compromise of the limb, thus leading to long term loss of nerve and/or muscle function. Other factors that may affect a physician's choice of treatment include associated injuries, skeletal maturity, comorbidity, etc. It is the clinical input from physician's experience dealing with cases and the recorded outcomes that helps increase the probability of rightfully identifying patients who will benefit from a particular treatment option $^{6}$. The aim of this study was to reveal the treatment outcome of children with extension type Gartland type II and type III supracondylar fracture of the humerus who were treated by closed reduction and internal fixation using percutaneous crossed k-wires.

\section{MATERIALS AND METHODS}

This was a prospective observational study conducted in Chattogram, Bangladesh, between January 2018 to September 2020. A total of 70 children with either Gartland type II or type III supracondylar fracture of the humerus were enrolled as study subjects from two different hospitals. They were all treated by closed reduction and internal fixation using percutaneous crossed k-wires. Inclusion criteria for study was ages between 2-14 years diagnosed with Gartland type II or type III supracondylar extension type fracture of the humerus. Patients with open fractures, with Gartland type I fractures and the rare form of flexion type supracondylar fractures were excluded from this study. After obtaining informed written consents from their respective guardians the children were enrolled in the study.

\section{Treatment procedure}

i) The patient was positioned supine with the affected extremity on a fluoroscopy detector or hand-table.

ii) Closed reduction was performed in the following method :-

- Longitudinal traction was applied with the elbow in $30^{\circ}$ of flexion.

- Medial or lateral translational displacement was corrected.

- Varus or valgus malalignment was corrected.

- Traction was maintained and elbow was flexed, placing pressure over the olecranon process to correct extension at the fracture site.

iii) Reduction was assessed using anteroposterior, oblique and lateral fluoroscopic views for good functional outcome. iv) Kirschner wires of size 1.5-2 $\mathrm{mm}$ for younger children and 2-2.5 $\mathrm{mm}$ for older children were used for fixation. Prior to insertion of the wires, the medial and lateral condyles were identified using image intensifier and the direction and length of the wires were assessed.

v) With postero-medial displacement, the arm was placed in maximum external rotation on platform and the medial pin was inserted first. To avoid ulnar nerve injury, the left thumb was placed behind the medial epicondyle in case of the right limb, while the left index finger was placed behind medial epicondyle in case of left limb. With postero-lateral displacement, the arm was placed in maximum internal rotation on the platform and the lateral pin was inserted first.

vi) Stability was assessed by moving the elbow through a range of motion under live fluoroscopy in the lateral projection.

vii) The pins were cut and bent outside the skin. Then a wellpadded dressing was applied and movement was initiated as early as 24 hours after operation.

The pins were removed between 3 and 4 weeks post operatively depending on patient age and stability of fracture. Radiological union was evaluated and functional outcome of this treatment was determined using the Flynn criteria as in the table below.

Table I : Flynn's Criteria

\begin{tabular}{llcc} 
Result & Rating & $\begin{array}{c}\text { Functional outcome } \\
\text { (Motion loss) }\end{array}$ & $\begin{array}{c}\text { Cosmetic factor } \\
\text { (Loss of carrying angle) }\end{array}$ \\
Satisfactory & Excellent & $0-5$ & $0-5$ \\
& Good & $5-10$ & $5-10$ \\
Unsatisfactory & Fair & $10-15$ & $10-15$ \\
& Poor & $>15$ & $>15$ \\
\hline
\end{tabular}

\section{RESULTS}

A total of 70 children were enrolled in the study. The patient demographics are illustrated in the table below.

Table II : Baseline characteristics of study subjects

\begin{tabular}{lr} 
Baseline characteristics & \\
Age & $8.14 \pm 2.8$ \\
Gender (Male/Female) & $43 / 27$ \\
Arm involved (Left/ Right) & $27 / 43$ \\
\hline
\end{tabular}

The male to female ratio was $1.6: 1$ with 43 male and 27 female respondents. Their ages were between 2 to 13 years old with mean age of 8.14 years. The left arm was involved in $27(38.6 \%)$ children and the right arm was involved in 43(61.4\%). In all study subjects the right hand was the dominant hand.

Table III : Complications observed among study subjects

Complications

Frequency

Preoperative

Radial nerve injury 1

Pulseless pink hand 4

Post-operative

S.P.T. I

5

U.N neuropraxia 
Among pre-operative complications, one patient had radial nerve injury and three had pulseless pink hand. Radiological union was observed in all patients at 3 weeks. Amidst postoperative complications $5(7.1 \%)$ children showed Superficial Pin Tract Infection (S.P.T.I) and 1(1.4\%) child had ulnar nerve neuropraxia.

Table IV : Radiographic and clinical outcome of study subjects $(\mathrm{n}=70)$

\begin{tabular}{lrrr} 
& Uninjured elbow & Injured elbow & p-value \\
Carrying angle & 10.37 & 8.39 & $<0.001$ \\
Elbow Flexion & 134.86 & 130.67 & $<0.001$ \\
Elbow Extension & -0.27 & 3.41 & $<0.001$ \\
\hline
\end{tabular}

Significant difference was observed in both radiographic and clinical outcome of the study subjects when the injured arm was compared with the uninjured arm.

However, when the outcomes were categorized according to the criteria suggested by Flynn et al, the results were as shown in the table below ${ }^{7}$.

Table V : Outcome according to Flynn's criteria

\begin{tabular}{lrrr} 
Flynn's Criteria & Overall Grade & $\begin{array}{r}\text { Carrying angle } \\
\text { change(n=70) }\end{array}$ & $\begin{array}{r}\text { ROM Deficit } \\
(\mathbf{n}=\mathbf{7 0})\end{array}$ \\
Excellent & $0-5$ & 57 & 35 \\
Good & $6-10$ & 10 & 22 \\
Fair & $11-15$ & 3 & 5 \\
Poor & $>15$ or cubitus varus & 0 & 8 \\
\hline
\end{tabular}

When looking at the cosmetic factor, changes between 0-5 degrees carrying angle was seen in 57 children, while 10 children showed a change between 6-10 degrees. Only 3 children showed a change of carrying angle between 11-15 degrees. No child had a carrying angle of more than 15 degrees. In contrast, the functional factor was significantly different from the carrying angle change with only 35 children showing minimal deficit, and 8 children showed poor outcome with a $>15^{\circ}$ loss of flexion /extension, the highest being a loss of $45^{\circ}$ in one child.

Table VI : Clinical results compared with those of other literatures for carrying angle loss

\begin{tabular}{|c|c|c|c|c|c|}
\hline \multirow[t]{2}{*}{ Authors } & \multirow{2}{*}{$\begin{array}{l}\text { Total number } \\
\text { of cases }\end{array}$} & \multicolumn{4}{|c|}{ Flynn's criteria (Carrying angle loss) } \\
\hline & & Excellent & Good & Fair & Poor \\
\hline Foead et $\mathrm{al}^{8}$ & 28 & 21 & 4 & 2 & 1 \\
\hline Mehserle and Meehan ${ }^{9}$ & 33 & 23 & 7 & 1 & 2 \\
\hline Ehsan et al ${ }^{*}$ & 70 & 57 & 10 & 3 & 0 \\
\hline
\end{tabular}

*This study.

When compared to other similar studies, the cosmetic outcome in our study outshone them with no study showing a poor outcome.
Table VII : Clinical results compared with those of other literatures for functional loss

\begin{tabular}{|c|c|c|c|c|c|}
\hline \multirow[t]{2}{*}{ Authors } & \multirow[t]{2}{*}{ Total number of cases } & \multicolumn{4}{|c|}{ Flynn's criteria (ROM deficit) } \\
\hline & & Excellent & Good & Fair & Poor \\
\hline Gupta et al ${ }^{10}$ & 25 & 18 & 7 & 0 & 0 \\
\hline Julfiqar et al ${ }^{11}$ & 35 & 24 & 6 & 3 & 2 \\
\hline Ehsan et $\mathrm{al}^{*}$ & 70 & 35 & 22 & 5 & 8 \\
\hline
\end{tabular}

*This study.

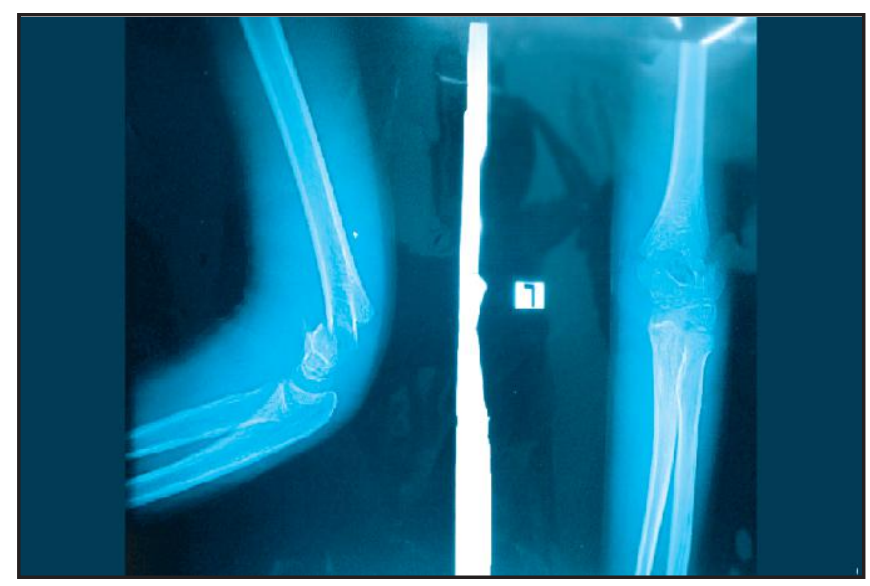

Figure 1 a) : Pre operative X-ray

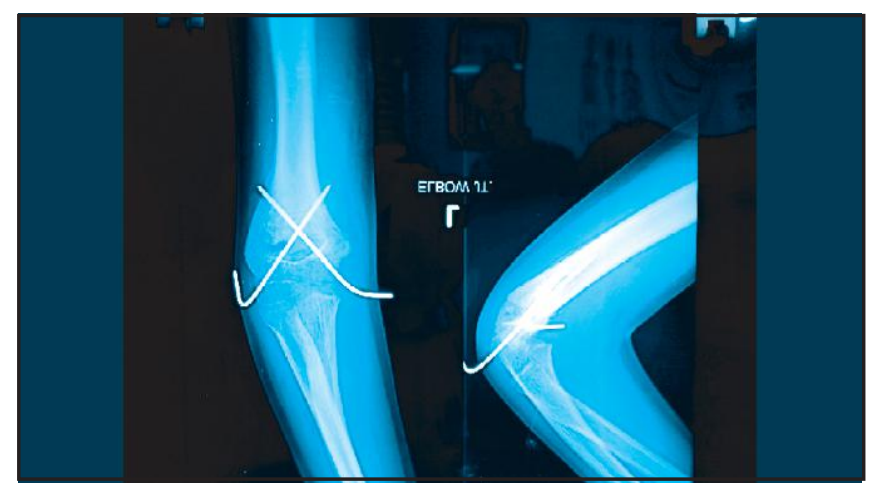

Figure 1 b) : 3 weeks post operative X-ray shows radiological signs of union

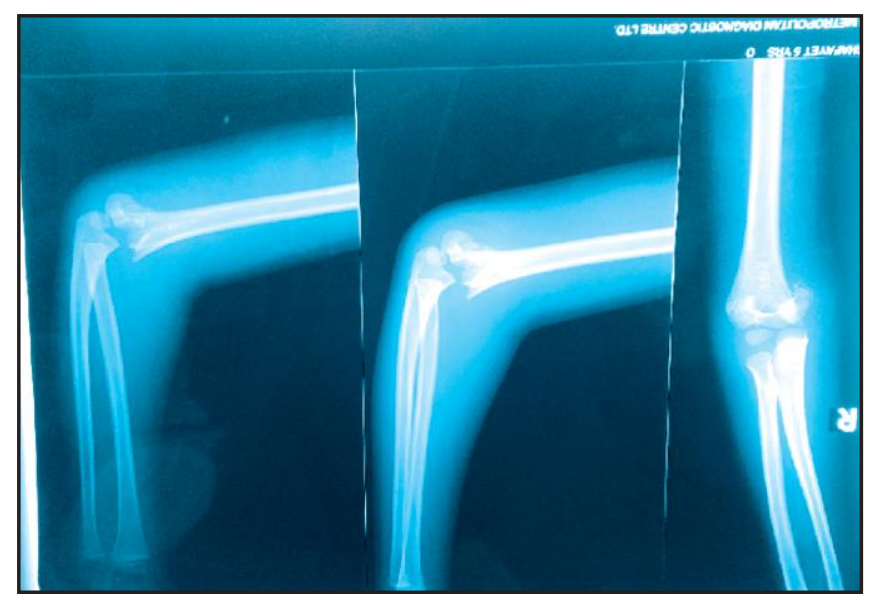

Figure 2 a) : Pre operative X-ray 


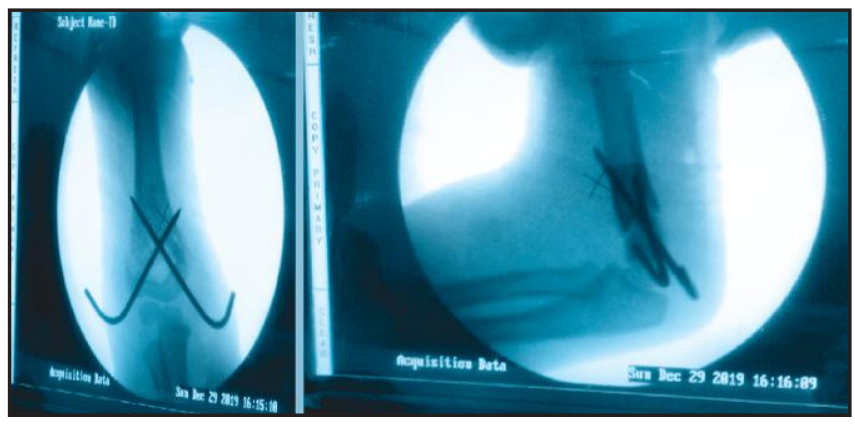

Figure 2 b) : Immediate post operative intensifier view both in $\mathrm{AP}$ and Lateral view

\section{DISCUSSION}

A lot of attention is directed towards the outcome in children ensuing treatment for a particular type of fracture. In case of supracondylar fractures of the humerus, the focus lies on obtaining a reduction as close to anatomical position as possible so as to prevent cubitus varus or valgus in the future. Although Type I fractures can be successfully treated with closed reduction and external fixation using a plaster cast, the same is impossible in type II and type III fractures where maintaining the reduced fracture would definitely need some form of internal fixation.

Since closed reduction with percutaneous pinning is the golden standard for treating such fractures many studies were found using this technique ${ }^{1}$. However, there was a variation found in the location of pins for fixation. Foead et al compared two different methods of percutaneous pin fixation namely medial lateral pin fixation and two lateral pin fixations ${ }^{8}$. Fahmy et al compared posterior intrafocal pinning with other types of pinning methods such as lateral pinning, crossed wires, two lateral and one medial wire etc ${ }^{12}$. Hussain compared two different procedures for percutaneous K-wire fixation, namely two lateral parallel pin fixation vs cross medial lateral pin fixation $^{13}$.

In our study, crossed k- wires were used, one inserted from lateral side and one from the medial side. The outcome of the patients was excellent in more than $80 \%$ of the patients according to Flynn's criteria for changes in carrying angle, with 3 unsatisfactory results. A similar finding was observed in many other studies ${ }^{13,14}$.

However, only $35(50 \%)$ children had excellent functional ability categorized by Flynn criteria that was based on range of motion deficit between the normal versus injured arm. Additionally, 13(18.6\%) children had unsatisfactory results with a high variation between the injured and uninjured arm. The overall mean carrying angle, elbow flexion and elbow extension showed a significant difference between the injured and the uninjured arms. This implies that despite majority of patients having an excellent cosmetic result and an overall good functional result, the injured arm was still not as good as the uninjured arm.
Compared to this, a 2016 study by Alam et al adopted an anterior joystick technique with Schantz pins for fixation in unstable supracondylar fractures with rotational deformity ${ }^{15}$. In that study, excellent functional outcome for range of motion was observed in 31 out of the 36 patients. Furthermore, no significant difference was observed between the mean carrying angle, flexion and extension between the injured and uninjured arm. Since the number of children in our study is almost twice the number in the above-mentioned study and hence more reliable, inability to obtain a non-significant difference between injured and normal arm is plausible. In fact, most of the studies had a small sample size with hardly 40 cases and this could be a factor for variation in results ${ }^{10,11,13}$. Again, the follow up duration to assess patient's range of motion were comparatively shorter than the study by Alam. If follow up for a longer time duration could have been done, the outcome may have been even better. Nevertheless, another study by Hussein showed satisfactory functional out come in $80 \%$ of the cases, a finding consistent with our study ${ }^{13}$.

One major complication of using cross K-wire fixation is iatrogenic ulnar nerve injury. In our study, only one developed ulnar nerve neuropraxia where as five others $(7 \%)$ developed superficial pin tract infections. The results were higher in some studies and lower in others ${ }^{16,13}$. Although the risk of peripheral nerve injury ranges between $2-6 \%$ in various studies, our study found a risk of only $1.42 \%(n=1)$.

Fortunately, no vascular injury nor compartment syndrome was observed postoperatively since treatment was done within 72 hours of admission hence avoiding swelling and other complications that make closed reduction almost impossible. In all the patients, movement of the injured limb was initiated after 24 hours of surgery to limit stiffness of the limb. No adverse effects were observed due to this.

\section{CONCLUSION}

Treatment of extension type fractures using crossed k-wires provides a better cosmetic benefit than functional recovery in majority of the patients. Methods to further modify this technique should be looked in to so as to improve the functional recovery even more. Along with this a prolonged duration of follow up can yield better and more accurate results.

\section{DISCLOSURE}

All the authors declared no competing interest. 


\section{REFERENCES}

1. Vaquero-Picado A, González-Morán G, Moraleda L. Management of supracondylar fractures of the humerus in children. EFORT open reviews. 2018;3(10):526-540.

2. Turhal O, K na M, Karaduman ZO, Turhan Y, Kaya O, Güler C. Temporary Fixation of Reduction with Fabric Adhesive Bandage in the Surgical Treatment of Pediatric Supracondylar Humerus Fractures. Medicina. 2019;55(8):450.

3. Mallo G, Stanat SJ, Gaffney J. Use of the Gartland classification system for treatment of pediatric supracondylar humerus fractures. Orthopedics. 2010;33(1).

4. Lustosa L \& Gaillard F; 2020. Radiopedia. [Online].

Available at: https://radiopaedia.org/articles/gartland-classification-of-supracondylar-humeral-fractures. [Accessed 16 November 2020].

5. Uzzaman KS, Awal KA, Alam MK. Closed Reduction and Percutaneous Kirschner Wire Fixation Combined with Plaster Cast versus Conventional Plaster Cast immobilization in the Treatment of Colles' Fracture A Prospective Randomized Comparative Study. Journal of Dhaka Medical College. 2008;17(2):98-105.

6. Howard A, Mulpuri K, Abel MF, Braun S, Bueche M, Epps H, Hosalkar H, Mehlman CT, Scherl S, Goldberg M, Turkelson CM. The treatment of pediatric supracondylar humerus fractures. JAAOS-Journal of the American Academy of Orthopaedic Surgeons. 2012;20(5):320-327.

7. Flynn JC, Matthews JG, Benoit RL. Blind pinning of displaced supracondylar fractures of the humerus in children. Sixteen years' experience with long-term follow-up. J Bone Joint Surg [Am]. 1974; 56:263-272.

8. Foead A, Penafort R, Saw A, Sengupta S. Comparison of two methods of percutaneous pin fixation in displaced supracondylar fractures of the humerus in children. Journal of orthopaedic surgery. 2004;12(1):76-82

9. Mehserle WL, Meehan PL. Treatment of the displaced supracondylar fracture of the humerus (Type III) with closed reduction and percutaneous cross-pin fixation. Journal of pediatric orthopedics. 1991;11(6):705-711.

10. Gupta HK, Khare KD, Chaurasia D. Results of lateral pin fixation for the displaced supracondylar fracture of humerus in children. Journal of College of Medical Sciences-Nepal. 2012;8(1):13-17.

11. Julfiqar M, Pant A, Huda N, Aslam M. Outcomes of Percutaneous Medial-Lateral Cross Pinning of the Pediatric Supracondylar Fractures of the Humerus. International Journal of Scientific Study. 2015;3(7):112-116.

12. Fahmy MA, Hatata MZ, Al-Seesi H. Posterior intrafocal pinning for extension-type supracondylar fractures of the humerus in children. The Journal of bone and joint surgery. British volume. 2009;91(9):1232-1236.

13. Hussein MT.Comparative study between two patterns of percutaneous K-wire fixation of supracondylar fracture of the humerus in children Muntansiriya Medical Journal. 2016;15(2): 14-19.

14. Boyd DW, Aronson DD. Supracondylar fractures of the humerus: a prospective study of percutaneous pinning. Journal of Pediatric Orthopaedics. 1992;12(6):789-794.

15. Alam QS, Sarwar MG, Hossain M, Rahman MA, Salam SI, Ariph GU, Mollah RA. A new method for closed reduction and percutaneous pinning in pediatric supracondylar humerus fracture with or without rotational malalignment those are difficult to reduce. J Orthop Truma 2019;33-35.

16. Aktekin CN, Toprak A, Ozturk AM, Altay M, Ozkurt B, Tabak AY. Open reduction via posterior triceps sparing approach in comparison with closed treatment of posteromedial displaced Gartland type III supracondylar humerus fractures. Journal of Pediatric Orthopaedics B. 2008;17(4):171-178. 